\title{
Holistic Twig Joins on Indexed XML Documents*
}

\author{
Haifeng Jiang Wei Wang Hongjun Lu \\ Dept. of Computer Science \\ The Hong Kong Univ. of Science and Technology \\ Hong Kong, China \\ Email: \{jianghf, fervvac, luhj\}@cs.ust.hk
}

\author{
Jeffrey $\mathrm{Xu} \mathrm{Yu}$ \\ Dept. of Systems Engineering \\ and Engineering Management \\ The Chinese Univ. of Hong Kong \\ Hong Kong, China \\ Email: yu@se.cuhk.edu.hk
}

\begin{abstract}
Finding all the occurrences of a twig pattern specified by a selection predicate on multiple elements in an XML document is a core operation for efficient evaluation of XML queries. Holistic twig join algorithms were proposed recently as an optimal solution when the twig pattern only involves ancestordescendant relationships. In this paper, we address the problem of efficient processing of holistic twig joins on all/partly indexed XML documents. In particular, we propose an algorithm that utilizes available indices on element sets. While it can be shown analytically that the proposed algorithm is as efficient as the existing state-of-the-art algorithms in terms of worst case $\mathrm{I} / \mathrm{O}$ and $\mathrm{CPU}$ cost, experimental results on various datasets indicate that the proposed index-based algorithm performs significantly better than the existing ones, especially when binary structural joins in the twig pattern have varying join selectivities.
\end{abstract}

\section{Introduction}

$\mathrm{XML}$ is emerging as a de facto standard for information exchange over the Internet. Although XML

The project was partly supported by the Research Grant Council of the Hong Kong SAR, China (Grants AoE/E-01/99 and HKUST6060/00E) and National 973 Fundamental Research Program of China (G1998030414).

Permission to copy without fee all or part of this material is granted provided that the copies are not made or distributed for direct commercial advantage, the VLDB copyright notice and the title of the publication and its date appear, and notice is given that copying is by permission of the Very Large Data Base Endowment. To copy otherwise, or to republish, requires a fee and/or special permission from the Endowment.

Proceedings of the 29th VLDB Conference, Berlin, Germany, 2003 documents could have rather complex internal structures, they can generally be modelled as ordered trees. Queries in XML query languages (see, e.g., [1, 2]) typically specify patterns of selection predicates on multiple elements which have some specified structural relationships. For example, to retrieve all paragraphs that are nested inside sections and have at least one figure and one table can be expressed as

\section{//section//paragraph[figure AND table]}

Such a query can be represented as a node-labelled twig pattern (or a small tree) with elements and string values as node labels [3].

Finding all occurrences of a twig pattern is a core operation in XML query processing [6, 13, 10, 15]. A typical approach is to first decompose the pattern into a set of binary structural relationships (parentchild or ancestor-descendant) between pairs of nodes, then match each of the binary structural relationships against the XML database and finally stitch together the results from those basic matches [20, 9, 14, 4, 8, 19]. For example, to answer the twig pattern above, we first retrieve all the section, paragraph, figure and table element sets, possibly through a tag index. A possible evaluation strategy works as follows: (1) finding all (paragraph, figure) and (paragraph, table) pairs with two separate structural joins; (2) merging these results to obtain the paragraphs with a figure and a table; and (3) joining these paragraphs with all sections through another structural join.

The main disadvantage of such a decomposition based approach is that intermediate result sizes can get very large, even when the input and the final result sizes are much more manageable. To address the problem, Bruno et al proposed a holistic twig join approach for matching XML query twig patterns [3]. With a chain of linked stacks to compactly represent partial results of individual query root-to-leaf paths, their approach merges the sorted lists of participating element sets altogether, without creating large intermediate results. 
The work reported in this paper is motivated by the following observations: Although the proposed holistic twig join algorithm has been proved to be I/O and CPU optimal in terms of input and output sizes for twigs with only ancestor-descendant edges (and yet still efficient for patterns with parent-child edges), the potential benefit of skipping elements that do not participate in a final twig match by using available indices is not fully explored. In our previous study on structural joins using indices [8], we found that the benefit of skipping elements without matches could be enormous when the input lists are large while few of the elements appear in the output.

In this paper, we address the problem of efficient holistic processing of twig joins on indexed XML documents. In particular, we propose a generic algorithm, TSGeneric $^{+}$, which can utilize available indices, e.g. XR-trees [8], on element sets. The main feature of TSGeneric $^{+}$is that it uses indices to quickly locate the first match for a sub twig pattern (starting from the current elements of the input lists in the sub pattern). Locating the first match for a sub twig pattern can be evaluated by "fixing" edges that do not comply with the desired structural relationship (e.g., ancestordescendant relationship) with a structural join like algorithm. The main issue here is which edge to choose first so that more elements without matches can be skipped. We propose three edge-picking heuristics in this paper: top-down, bottom-up and statistics-based, and study their performance with our experiments in comparison with existing algorithms.

Our contributions can be summarized as follows:

1. We propose a general holistic twig join processing algorithm, namely TSGeneric, which makes use of a set of stacks to cache elements and a cursor interface that provides standard methods to return elements with possible matches. With different implementations of the cursor interface, algorithms can be developed to process twig joins based on available access methods.

2. We propose the TSGeneric ${ }^{+}$algorithm based on TSGeneric to exploit more opportunities to skip elements. In particular, three different heuristics, top-down, bottom-up and statistics-based, are proposed to select the first edge to start the processing. As such, the potential of skipping elements with various indices is further exploited.

3. An extensive performance study with datasets of various characteristics was conducted. Our results show that the TSGeneric ${ }^{+}$algorithm on the XR-tree indexed data (regardless of the edgepicking heuristics used) significantly outperforms the existing algorithms, namely TwigStack and its variant TwigStackXB, in terms of various evaluation metrics. While among the three edge-picking heuristics, the statistics-based heuristic is most robust.

The rest of the paper proceeds as follows. Section 2 is dedicated to some background knowledge and related work on XML. We present the TSGeneric algorithm in section 3 . Then we proceed to present the TSGeneric ${ }^{+}$algorithm and its worst case performance analysis in section 4 . Section 5 reports experimental results. Section 6 concludes the paper.

\section{Background and related work}

\subsection{Data model and numbering schemes}

XML data is commonly modelled by a tree structure, where nodes represent elements, attributes and text data, and parent-child pairs represent nesting between XML element nodes. Most existing XML query processing algorithms rely on a positional representation of element nodes, where each element is represented with a tuple of three fields: (start, end, level $)^{1}$, based on its position in the data tree $[20,14,4,8]$. Such a numbering scheme is also called as region encoding. Formally, element $u$ is an ancestor of element $v$ iff $u . s t a r t<v$.start $<$ u.end. For parent-child relationship, we also test whether $u$.level $=$ v.level -1 .

Corollary 1 Given two elements $e_{i}$ and $e_{j}$, if $e_{i}$. start $<e_{j}$.start and $e_{i}$ is not an ancestor of $e_{j}$, then $e_{i}$ will not be an ancestor of any element $e_{x}$ with $e_{x}$. start $>e_{j}$. start.

Figure 1 shows a fictitious XML document which contains a root and other elements with tag $a$ or $b$ or $c$. The region encoding is also shown for each element.

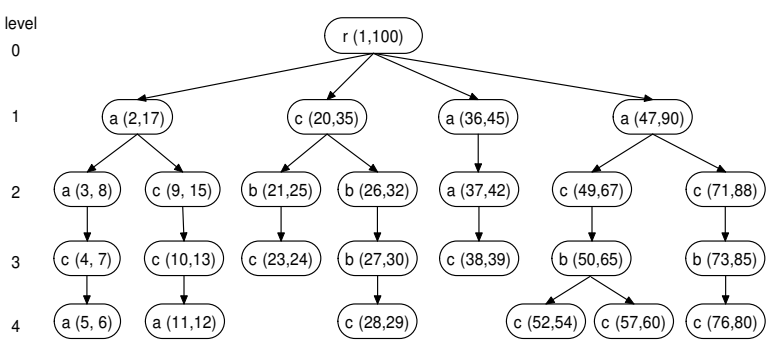

Figure 1: A sample XML document

\section{$2.2 \quad$ Structural joins}

A structural join is to find all occurrences of a given structural relationship between two sets of elements. A series of structural join algorithms were

\footnotetext{
${ }^{1}$ In an XML database with multiple XML documents, we may also need to record which document an element belongs to with an additional DocId field. Although we do not consider the DocId field in our algorithms, they can be easily extended to support DocId.
} 
proposed in the literature. Merge-based algorithms include MPMGJN [20], $\mathcal{E} \mathcal{E} / \mathcal{E} \mathcal{A}$-Join [9] and Stack-TreeDesc/Anc [14]. [4, 7, 8] are index-based approaches. In particular, XR-tree was recently proposed to index XML data to support efficient structural joins [8]. The experimental results showed that the XR-tree based algorithm, namely, XR-stack, performs the best among all existing structural join algorithms, especially when the join selectivity of (at least one of) the participating element sets is high [8] (i.e. few matched elements).

\subsection{Twig pattern matching}

A twig pattern is a selection predicate on multiple elements in an XML document. Such query patterns can generally be represented as node-labelled trees. Matching a twig pattern against an XML database is to find all occurrences of the pattern in the database. Formally, given a query twig pattern $Q$ and an XML database $D$, a match of $Q$ in $D$ is identified by a mapping from nodes in $Q$ to nodes in $D$, such that: (i) query node predicates are satisfied by the corresponding database nodes; (ii) the structural (parent-child or ancestor-descendant) relationships between query nodes are satisfied by the corresponding database nodes. The answer to $Q$ with $n$ nodes can be represented as an $n$-array relation where each tuple $\left(d_{1}, d_{2}, \cdots, d_{n}\right)$ consists of the database nodes that identify a distinct match of $Q$ in $D$.

The most related work on twig pattern matching is a merge-based holistic twig join algorithm proposed in [3]. The recent work by $\mathrm{Wu}$ et al [19] emphasized on join order selection when a twig pattern is evaluated with the traditional decomposition approach.

\section{The generic twig join algorithm}

In this section, we first introduce a general setting of the twig join problem, followed by the description of a generic twig join framework, TSGeneric. Then we discuss how such generic work can be seamlessly extended to the case when element sets participating in the twig join are indexed.

\subsection{Preliminaries}

A twig pattern can be represented with a tree. The self-explaining functions isRoot $(q)$ and isLeaf $(q)$ examine whether a query node $q$ is a root or a leaf node. The function children $(q)$ gets all child nodes and parent $(q)$ returns the parent node of $q$. The function subtreeNodes $(q)$ returns node $q$ and all its descendants. When there is no ambiguity, we may also refer to node $q$ as the sub query tree rooted at $q$. In the rest of the paper, "node" refers to a tree node in the twig pattern (e.g., node $q$ ), while "element" refers to the elements in the dataset involved in a twig join.

We assume there is a data stream associated with each node in the query tree. Every element in the data stream is already encoded in the following region format: (start, end, level). Each data stream is already sorted on the start attribute.

We also assume the join algorithms will make use of two types of data structures: cursors and stacks. Given a query tree $T$, we associate a cursor $\left(C_{q}\right)$ and a stack $\left(S_{q}\right)$ to every node $q \in T$, as shown in Figure 2 .

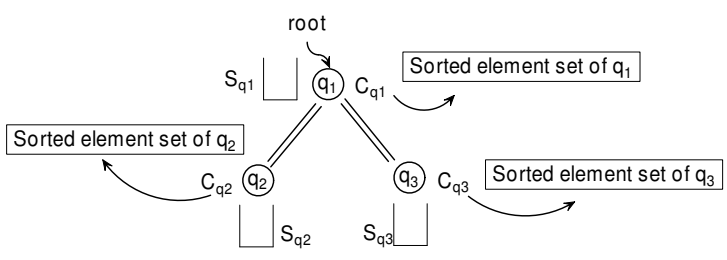

Figure 2: Cursors and stacks during execution

Each cursor $C_{q}$ points to some element in the corresponding data stream of node $q$. Henceforth, " $C_{q}$ " or "element $C_{q}$ " will refer to the element $C_{q}$ points to, when there is no ambiguity. The cursor can move to the element (if any) next to element $C_{q}$. Such behavior can be invoked with $C_{q} \rightarrow$ advance(). Similarly, we can access the attribute values of element $C_{q}$ by $C_{q} \rightarrow$ start, $C_{q} \rightarrow$ end and $C_{q} \rightarrow$ level. Initially, all the cursors point to the first element of the corresponding data stream.

Initially, all stacks are empty. During query execution, each stack $S_{q}$ may cache some elements before the cursor $C_{q}$ and these elements are strictly nested from bottom to top, i.e. each element is a descendant of the element below it. We also associate with each element $e$ in $S_{q}$ a pointer to the lowest ancestor in $S_{\text {parent }(q)}$. Thus, we can efficiently access all $e$ 's ancestors in $S_{\text {parent }(q)}$. In fact, cached elements in stacks represent the partial results that could be further extended to full results as the algorithm goes on.

Next, we define an important concept, which is key to the understanding of the TSGeneric algorithm.

Definition 1 (Solution Extension) We say that a node $q$ has a solution extension if there is a solution for the sub query rooted at $q$ composed entirely of the cursor elements of the query nodes in the sub query.

Note that, if node $q$ has a solution extension, since $C_{q}$ is the ancestor of all cursor elements in the sub query tree nodes, $C_{q} \rightarrow$ start is smaller than all cursor start values of query nodes in the subtree $q$, based on the strictly nested property of XML data.

\subsection{The generic twig join algorithm}

Here, we briefly introduce algorithm TSGeneric, which is partly inspired by algorithm TwigStack proposed in [3]. The algorithm is shown in Algorithm 1.

getNext(q) returns a query node $q_{x}$ in the subtree $q$, such that the following three criteria are met: (a) node $q_{x}$ has a solution extension; and (b) if $q_{x}$ has siblings, 

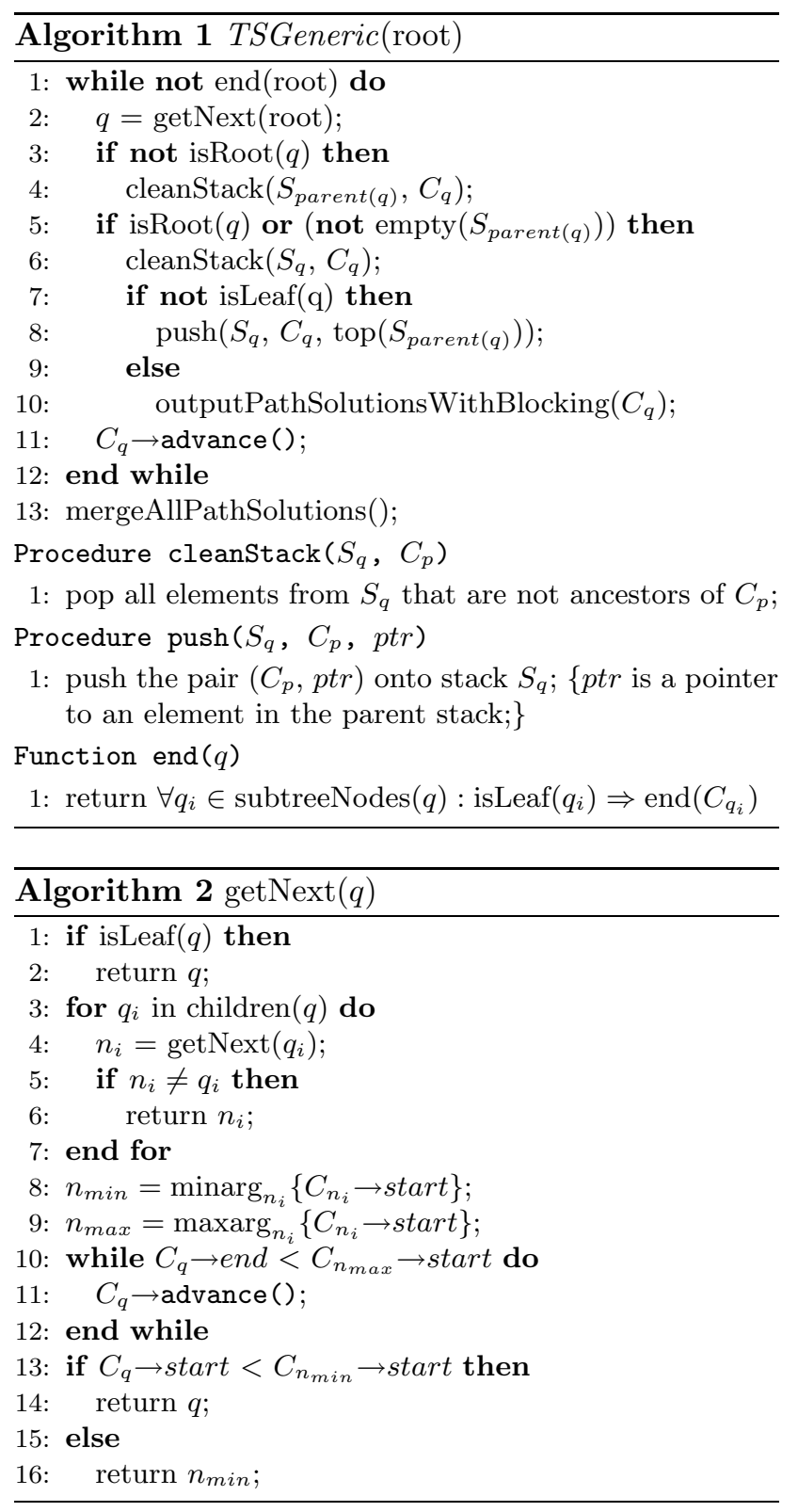

then $C_{q_{x}} \rightarrow$ start $<C_{q_{s}} \rightarrow$ start, where $q_{s}$ is a sibling of $q_{x}$ (note that all $q_{x}$ 's siblings must have a solution extension, otherwise, a lower query node would have been returned through line 5-6 in the algorithm); and (c) if $q_{x} \neq q, C_{\text {parent }\left(q_{x}\right)} \rightarrow$ start $>C_{q_{x}} \rightarrow$ start.

The key to getNext is to apply Corollary 1 . Called with the root of the query tree, getNext first traverses down to the left-most leaf node (by self recursive calls). Starting from the leaf node, it tries to find the highest possible query node with a solution extension by applying Corollary 1 (line 8-16). Given that all children have their own solution extensions (after line 7), in order for node $q$ to be returned, we make sure that node $q$ has a solution extension as well by advancing $C_{q}$ (line 11). If no common ancestor for all $C_{n_{i}}$ is found in $q$, we return the child node with the smallest start value (fulfilling criterion (b)), i.e. $n_{\min }$. Note that as long

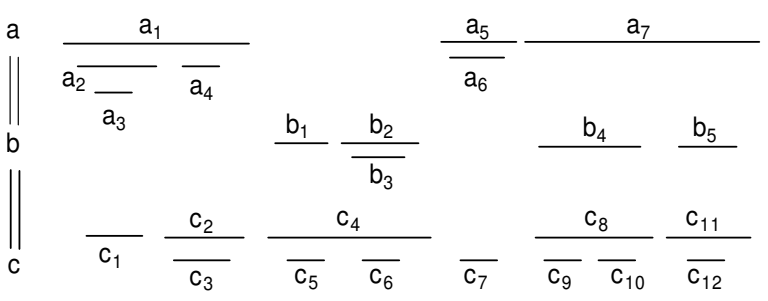

Figure 3: Query //a//b//c and element sets from the example document in Figure 1

as line 16 is executed and $n_{\min }$ is returned, the outer recursive calls to getNext will return the same node $n_{\min }$ all the way up through line 5-6.

Example 1 Consider a path query //a//b//c on the element sets visualized in Figure 3. A subscript is added to each element in the order of their start values for easy reference. Initially, the three cursors are $\left(a_{1}, b_{1}, c_{1}\right)$. The first four calls of getNext(root) will always return node $c$ (with cursors at $c_{1}$ to $c_{4}$ one by one) because none of elements $c_{1}$ to $c_{4}$ has an ancestor in set $b$. These $c$ elements are consumed by the caller TSGeneric. Right before the fifth call, the cursors are $\left(a_{1}, b_{1}, c_{5}\right)$. The next call of getNext(root) will return node $b$, whose solution extension is $\left(b_{1}, c_{5}\right)$. In addition, the cursor of node a will be forwarded to $a_{5}$, the one after $b_{1}$. After several calls of getNext(root), we will eventually reach a cursor setup $\left(a_{7}, b_{4}, c_{9}\right)$, which is actually the first match of the query.

It is not difficult to understand TSGeneric with the knowledge on how getNext works. In line 2, we retrieve the next node $q$ to process with getNext(root). Some cached elements can be popped from $S_{\text {parent }(q)}$ (line 4) and $S_{q}$ (line 6) according to Corollary 1 . If $q$ is not a leaf node, we push element $C_{q}$ onto $S_{q}$ (line 8); otherwise, all the path solutions involving $C_{q}$ can be output (line 10). Note that path solutions should be output in root-to-leaf order so that they can be easily merged together to form final twig matches (line 13). As a result, we may need to block some path solutions during output, similarly as done in Stack-Tree-Anc [14] and showSolutionsWithBlocking [3].

\subsection{Cursor interface for TSGeneric}

Recently, there have been many proposals that advocate building certain indices on XML data to accelerate query processing $[4,7,8,3]$. A natural question that follows is "Can we accelerate twig join processing in TSGeneric by taking advantage of available indices?".

Our answer to this question is affirmative. Briefly speaking, our solution is to extend the existing cursor interface to reflect new abilities to access elements through indices. In addition to the existing advance() method, we define two new methods: 
1. $C_{q} \rightarrow$ fwdBeyond $\left(C_{p}\right)$ forwards $C_{q}$ to the first element $e$, such that e.start $>C_{p} \rightarrow$ start.

2. $C_{q} \rightarrow$ fwdToAncestorOf $\left(C_{p}\right)$ forwards the cursor to the first ancestor of $C_{p}$ and returns TRUE. If no such ancestor exists, it stops at the first element $e$, such that e.start $>C_{p} \rightarrow$ start, and returns FALSE.

The detailed discussion on various implementation issues of these methods will be presented in section 4.2. For now, the readers can simply assume the cursor interface as a black box.

With the additional methods, we can have an improved version of the TSGeneric algorithm. In fact, we only need to change the getNext implementation, which is now named to getNextCursor (Algorithm 3).

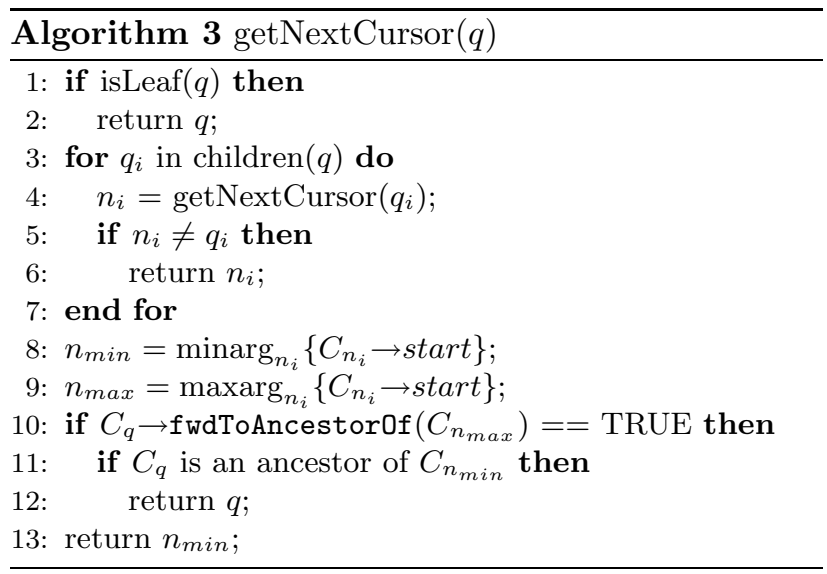

Line 10-13 in Algorithm 3 correspond to line 10-16 in Algorithm 2. The semantics is rather straightforward. If we find a common ancestor for all child cursors, we return $q$; otherwise, we return the child node with minimum start value, i.e. $n_{\min }$.

The benefit of the new getNextCursor call can be illustrated with the following example:

Example 2 Given the query //a//b//c and the element sets shown in Figure 3. Follow the example in Example 1. Right before the fifth call of getNextCursor (or getNext), the cursors are $\left(a_{1}, b_{1}, c_{5}\right)$. Although the next call of either getNextCursor or getNext will eventually forward the a's cursor to $a_{5}$, elements $a_{2}$ to $a_{4}$ need to be processed by getNext (line 10), while getNextCursor only needs to make a call of $C_{q} \rightarrow f w d T o A n c e s t o r O f\left(C_{n_{\max }}\right)$. As such, the processing time in getNextCursor is reduced.

Example 2 illustrates the following fact: if an index can efficiently support the new cursor methods, the new algorithm getNextCursor can avoid accessing many of the elements that do not contribute to final results but have to be scanned in getNext. On the other hand, it is still possible to implement those methods based on the advance() method (though inefficient).
The significance of the improved TSGeneric algorithm can be summarized as that we give an integrated and flexible solution:

- Integrated: we can deal with all-indexed datasets or partly-indexed datasets (To the best of our knowledge, no existing algorithm can achieve this).

- Flexible: our generic join framework can be used with any (or almost all existing) index for region coded XML datasets $\left(\mathrm{B}^{+}\right.$-tree, $\mathrm{R}$-tree, XB-tree and XR-tree).

\section{The TSGeneric ${ }^{+}$algorithm}

Although TSGeneric is able to skip some elements without matches through the generic cursor interface, the potential of skipping elements is in fact not fully exploited. In this section, we explore the advantage of skipping elements through the generic cursor interface with various heuristics.

Exploiting more benefit of skipping elements without matches relies heavily on a cursor-based structural join algorithm, namely, SJCursor, which is evaluated over edges of the query tree. The SJCursor algorithm (evaluated over an edge of the query tree) finds the first ancestor-descendant pair starting from the current cursors of the two nodes connected by the edge. Algorithm 4 shows the SJCursor algorithm. An edge $(p, c)$ is defined as "broken" if elements $C_{p}$ and $C_{c}$ do not have an ancestor-descendant relationship (see, function isBroken). SJCursor works as follows. If the edge is not broken, or either $C_{p}$ or $C_{c}$ reaches the end, it returns. Otherwise, if $C_{p} \rightarrow$ start is smaller than $C_{c} \rightarrow$ start, we call $C_{p} \rightarrow \mathrm{fwdToAncestor0f}\left(C_{c}\right)$ to move $C_{p}$ to the first ancestor element of $C_{c}$ (or beyond $C_{c}$ if no such ancestor exists); otherwise, we forward $C_{c}$ to the first element whose start value is larger than $C_{p} \rightarrow$ start, with $C_{c} \rightarrow \mathrm{fwdBeyond}\left(C_{p}\right)$, because a descendant element must have its start value larger than that of its ancestor element.

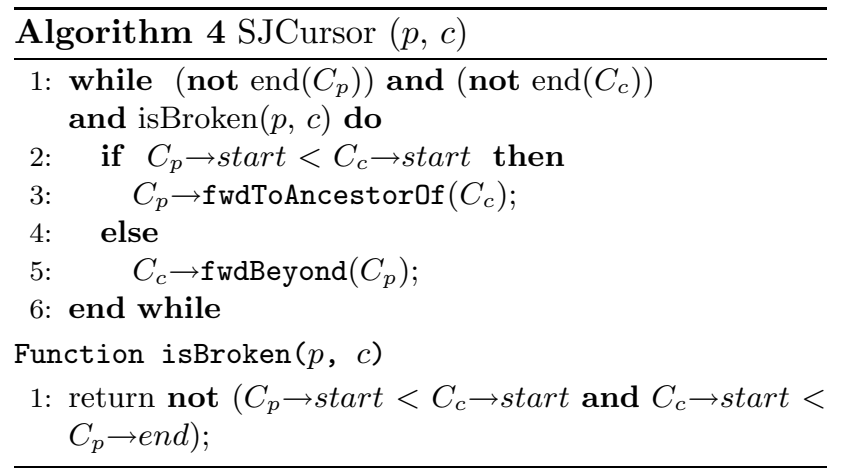

Example 3 Consider again the sample query in Figure 3. Suppose the current cursors are $\left(a_{1}, b_{1}, c_{1}\right)$. To find the first match for the query $/ / a / / b / / c$, which 
is $\left(a_{7}, b_{4}, c_{9}\right)$, a better evaluation strategy would be to first find the matching pair $\left(a_{7}, b_{4}\right)$ between node a and node $b$ by calling SJCursor( $a, b)$, and then call SJCursor $(b, c)$ to find the matching pair $\left(b_{4}, c_{9}\right)$. It is easy to verify that SJCursor $(a, b)$ only needs to access five elements, i.e. $a_{1}, b_{1}, a_{5}, b_{4}$ and $a_{7}$, while SJCursor $(b, c)$ only accesses elements $c_{1}$ and $c_{9}$. As a result, with this evaluation strategy, only 7 elements need to be processed, which is significantly better than the TSGeneric algorithm.

The challenge of such unordered evaluation as shown in Example 3 is that it might violate the correctness of the TSGeneric algorithm, because we may erroneously skip elements that do have matches. The following lemma is important to identify when such unordered evaluation is possible.

Lemma 1 Suppose a call of getNextCursor(root) returns a query node $q$. If the stack $S_{q_{a}}$ of any ancestor node $q_{a}$ of node $q$ is empty, then the current extension of node $q$ does not contribute to any further results and element $C_{q}$ can be discarded.

Proof There are two cases. The first case is that the ancestor node $q_{a}$ (whose stack is empty) is the parent of node $q$. Since $q \neq \operatorname{root}$ (otherwise, it could not have any ancestor), according to the criterion (c) of a query node returned by getNext, the start value of $C_{q}$ must be smaller than the start value of $C_{q_{a}}$ upon return. Given that $S_{q_{a}}$ is empty, it is clear that the extension of $q$ could not contribute to any new results. The second case is that $q_{a}$ is not the parent node of $q$. In other words, there exists some node $q^{\prime}$ which is the parent node of $q$ (obviously, $q_{a}$ is also an ancestor of $\left.q^{\prime}\right)^{2}$. Since $S_{q^{\prime}}$ is not empty, the extension of $q$ could possibly have an ancestor in $S_{q^{\prime}}$. We now prove that the elements in $S_{q^{\prime}}$ will eventually be popped without contributing to any further results. The reason is that for any element in $S_{q^{\prime}}$, all its ancestors in $q_{a}$ (if any) would have already been returned by previous calls of getNextCursor(root) and popped.

According to Lemma 1, if the stack of some node $q$ is empty, then, it is useless for getNextCursor(root) to return a node $q^{\prime}$ that is a descendant of $q$ in the query tree. In other words, as long as we discover the stack of a node $q$ is empty in the recursive call of getNextCursor, there is no need to further call getNextCursor for $q$ 's children. Rather, we should try to locate a solution extension for node $q$. Based on this, we improve getNextCursor by incorporating an extension-locating procedure, as shown in Algorithm 5. We name it as getNextExt. Lines 3-5 are newly added codes to getNextCursor: if the stack of node $q$ is empty, the procedure LocateExtension (Algorithm 6) is called, which

\footnotetext{
${ }^{2}$ Note that, according to algorithm TSGeneric, it is possible to have a node $q_{x}$ whose stack is not empty while the stack of some ancestor node of $q_{x}$ is empty because we do not pop stacks downward.
}

finds the first solution extension of node $q$, and then we simply return $q$. We call the TSGeneric algorithm TSGeneric $^{+}$if it calls getNextExt(root), other than getNextCursor(root).
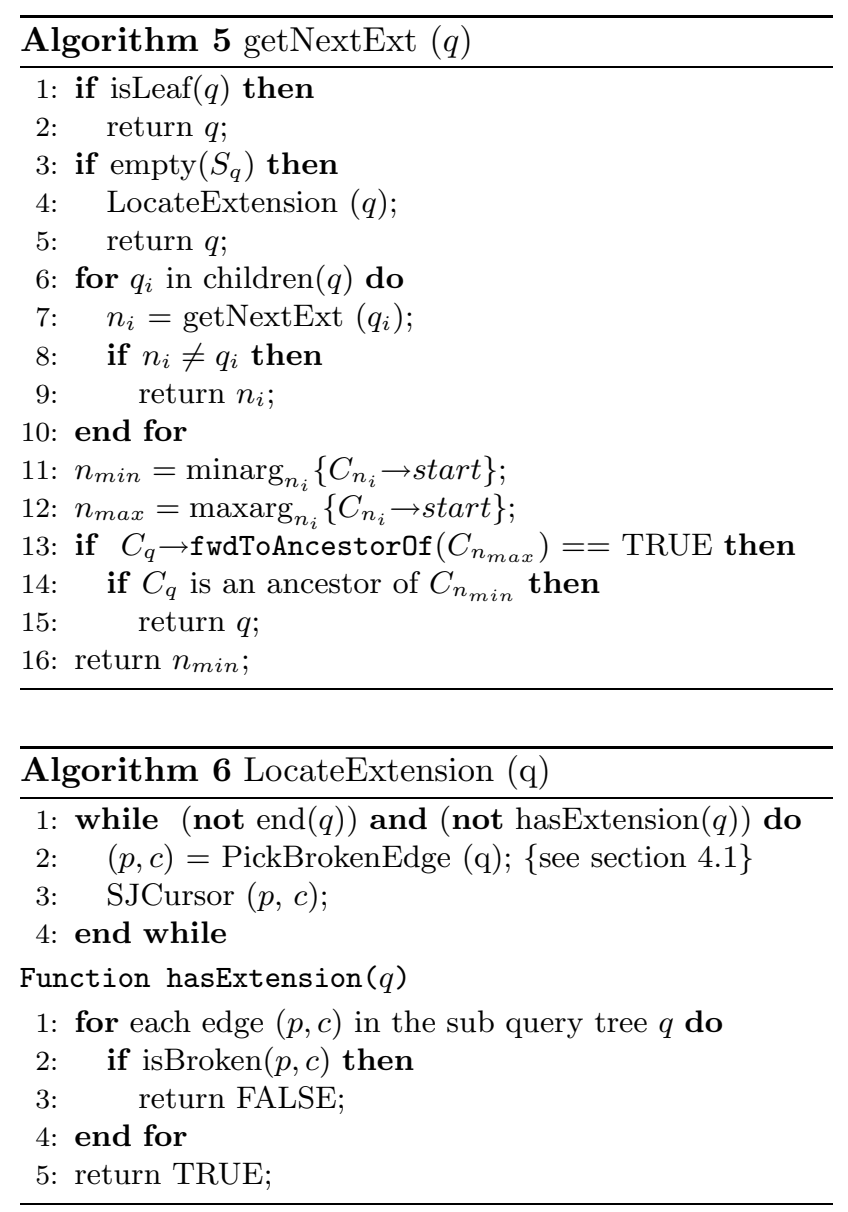

Consider the LocateExtension algorithm. It runs in a "pick-and-fix" fashion. Each time, it picks a broken edge (discussed in section 4.1) and fixes it with SJCursor, until node $q$ has a solution extension or any cursor in the subtree reaches the end. Note that the overhead to check for broken edges in function hasExtension is minimal because all the operations are carried out on the cursor elements of the query nodes and only negligible CPU cost is involved.

It is obvious that algorithm TSGeneric ${ }^{+}$, i.e. TSGeneric calling LocateExtension, works correctly and we have the following theorem (proof omitted in the interest of space):

Theorem 1 Given a query twig pattern $Q$ and an $X M L$ database $D$, the TSGeneric ${ }^{+}$algorithm correctly returns all answers for $Q$ on $D$.

\subsection{Heuristics for picking an edge}

Picking the next query edge to fix is essentially a query optimization problem. The optimization problem of join order selection has been extensively studied in the 
context of relational databases $[12,11,16]$. Due to the subtle difference in problem contexts, such previous work is not directly applicable to holistic twig joins considered here. In this subsection, we present some edge-picking heuristics.

Intuitively, we should choose an edge whose next match is the farthest from the current cursors of its two nodes, so that we can skip the most number of elements (without matches) when fixing other query edges. This has been illustrated in Example 3. We denote this heuristic as MD, reading "maximum distance".

The MD heuristic can leverage the work on structural join size estimation $[18,17]$. We now give the formula for estimating the average inter-match distance for a query edge $(p, c)$, whose two nodes are associated with element sets $p$ and $c$ respectively. Assume that we have statistics about the total number of elements in each set, i.e. $N_{p}$ and $N_{c}$, the width of the workspace for all regions in each element set, i.e. $W_{p}$ and $W_{c}$. If we can estimate the percentage $s_{p} \%$ of $p$ elements and the percentage $s_{c} \%$ of $c$ elements that have at least one match in the structural join between $p$ and $c$ (e.g., using histograms or sampling techniques proposed in [17]), then, the average distance between each match can be approximated as (assuming a uniform distribution):

$$
\operatorname{AvgDist}_{p \triangleleft c}=\min \left(\frac{W_{p}}{N_{p} \cdot s_{p} \%}, \frac{W_{c}}{N_{c} \cdot s_{c} \%}\right)
$$

Note that the two distance values estimated from set $p$ and set $c$ should be similar if elements in both sets are rarely nested. But the estimates might be different for highly nested datasets. We choose the smaller one.

Statistics might not always be available in realistic applications. We propose other two heuristics that work without assuming any knowledge about the element sets: top-down (TD) and bottom-up (BU).

Algorithm 7 shows the complete PickBrokenEdge algorithm. First, we retrieve all the broken edges in $q$ using a breadth first traversal order. The total number of broken edges is assumed to be $K$ (which is subject to change in different calls of PickBrokenEdge). Then, an edge is picked from the $K$ broken edges according to the heuristic specified by variable heuristic. Ties are broken arbitrarily in heuristic MD. By the top-down heuristic, we always choose the first broken edge. The deepest, right-most edge is chosen in the bottom-up heuristic.

\subsection{Cost analysis of TSGeneric ${ }^{+}$}

Although utilizing indices of various kinds built on element sets is expected to speed up the efficiency of the cursor interface to different extent, it is not clear whether the same optimal worst-case I/O and CPU

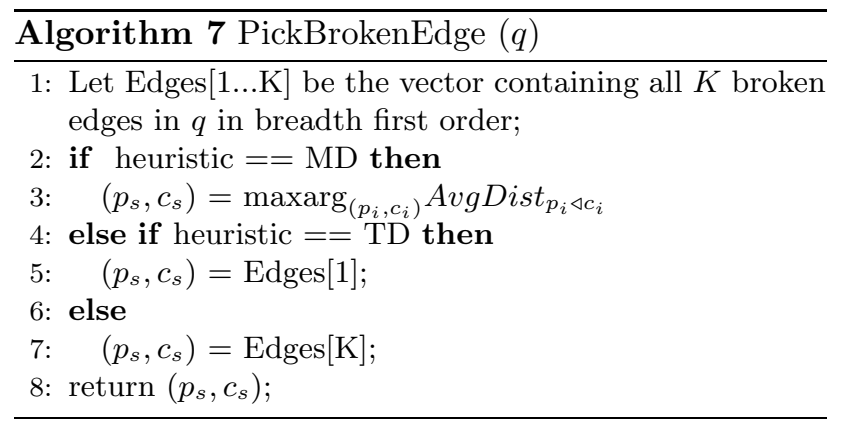

cost can be achieved when indices are used, compared to the TwigStack algorithm [3]. In this section, we address this problem.

Though, the effectiveness of different index structures varies, the worst-case $\mathrm{I} / \mathrm{O}$ and $\mathrm{CPU}$ cost for accessing elements through cursor interfaces built on them can be shown to be linear to the size of the elements indexed. The intuition is that each method of the cursor interface always drivers the cursor forward. To put it differently, the cursor never goes back. Therefore, it can be concluded that TSGeneric ${ }^{+}$that utilizes indices through cursor interfaces is as efficient as TwigStack in terms of worst-case I/O and CPU cost.

We focus ourselves on XR-tree as an example to show how a cursor interface based on indices can be implemented to achieve linear worst case I/O and CPU cost. The cursor interface implementation for other existing index structures should be similar. In the following, we first briefly introduce the structure of the XR-tree index, and then describe the details of cursor interface implementation based on XR-tree.

\subsubsection{The XR-tree index}

The XR-tree is an index structure recently proposed in [8] for indexing XML data based on the region encoding, i.e. (start, end, level). An XR-tree is basically a $\mathrm{B}^{+}$-tree (built on the start field of all indexed elements) augmented with stab lists and bookkeeping information in internal nodes. An element $e$ is included in the stab list of an index page $I$ if: (1) there exists some key $k_{i}$ in $I$ such that e.start $\leq k_{i} \leq$ e.end (or $k_{i}$ stabs the region of element $e$ ); and (2) no ancestor page of $I$ has a key that stabs $e$, i.e. $I$ is the highest index page that stabs $e$. Figure 4 shows the XR-tree for the set of $c$ elements in the example document in Figure 1. Note that element $(20,35,1)$ and $(49,67,2)$ are stabbed by index pages so that they are included in stab lists and also marked with "yes" in leaf pages.

Given an element $e$, searching for all its descendants in an element set $R$ indexed by an XR-tree is as simple as a $\mathrm{B}^{+}$-tree range search, i.e. e.start $<$R.start $<$ e.end. The novelty of XR-tree is that all the ancestors of $e$ can be collected from the stab lists of index pages and the leaf page when we navigate down the XR-tree using e.start (similar to a $\mathrm{B}^{+}$-tree equality search). 


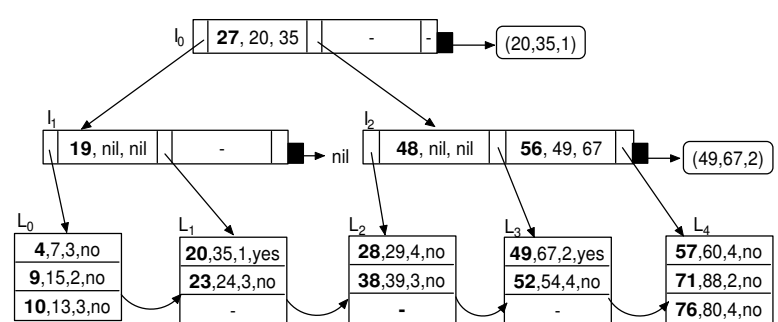

Figure 4: The XR-tree for $c$ elements in Figure 1

\subsubsection{Implementation of the cursor interface}

In our implementation, we keep in buffer the pages from root to leaf containing the cursor, denoted as hot path. For example, if the cursor is at element $(23,24,3)$ (Figure 4), then the hot path is $I_{0} \rightarrow I_{1} \rightarrow L_{1}$. Besides, we also keep the index entries followed to form the hot path. The current entry for page $I_{0}$ is -1 (the left-most entry), the entry for $I_{1}$ is 0 and the entry for $L_{1}$ is 1 . The entry information is useful to establish the linear CPU cost for index accesses.

To implement the $C_{q} \rightarrow$ advance() method, if $C_{q}$ is not the last element of the current leaf page, we simply points it to the next element without changing the hot path. Otherwise, we free the current leaf page and fetch in the next leaf page through the parent index page. If there is no more entry in the parent index page, we fetch in another index page through its parent similarly. For example, if the cursor is at $(9,15,2)$, to advance it, we simply move to the next element $(10,13,3)$. To advance again, we need to free $L_{0}$ and fetch in $L_{1}$. Note that the entry information of $I_{1}$ is also incremented. If the cursor is at $(23,24,3)$, we need to replace $I_{1}$ with $I_{2}$.

$C_{q} \rightarrow$ fwdBeyond $\left(C_{p}\right)$ is as simple as a $\mathrm{B}^{+}$-tree search. Starting from the current index entry of the root, sequentially scan the entries until the largest entry $k_{i}$, such that $k_{i} \leq C_{p} \rightarrow$ start is found. $k_{i}$.rightChild is the child page to be searched. If $k_{i}$.rightChild is different from the buffered page at the lower level, fetch in $k_{i}$.rightChild and replace the old page. The search keeps going on until we reach a leaf page. Then we set the cursor to the first element whose start is larger than $C_{p} \rightarrow$ start.

Consider the $C_{q} \rightarrow \mathrm{fwdToAncestor0f}\left(C_{p}\right)$ method. It is obvious that we do not need to touch any leaf pages or index pages (including their stab lists) that are before the hot path because what we want is the ancestor $e_{a}$ of $C_{p}$, such that $e_{a}$. start $\geq C_{q} \rightarrow$ start. If a qualified ancestor is found in some leaf page, the path from root to this leaf page would be the new hot path. If a qualified ancestor is found in some stab list of an index page, then the new hot path must include this newly accessed index page.

Theorem 2 In the TSGeneric ${ }^{+}$algorithm, the worst case $I / O$ and $C P U$ cost for accessing an element set $R$ through the cursor interface implemented on the $X R$ tree of set $R$ is linear to the size of $R$.

\subsection{Twig joins with parent-child edges}

Algorithm TSGeneric ${ }^{+}$can still be used to evaluate twig patterns with parent-child edges. The difference is that when we output a root-to-leaf path solution, we check the parent-child relationship using the level attribute of elements for parent-child edges. But the optimality in terms of worst case $\mathrm{I} / \mathrm{O}$ and $\mathrm{CPU}$ cost is no longer guaranteed. In particular, the algorithm might produce path solutions that do not contribute to any final match.

We use an example to illustrate the point. Consider a twig pattern //a [b]//c to be evaluated again the sample dataset in Figure 3. In the TSGeneric ${ }^{+}$ algorithm, the first call of getNextExt(root) will return node $a$ with cursors at $\left(a_{7}, b_{4}, c_{9}\right)$ (Recall that LocateExtension is called for root node $a$ since $S_{a}$ is empty initially). Then $a_{7}$ is pushed onto stack (line 7 in Algorithm 1). The next call of getNextExt(root) returns $b_{4}$. Since $b_{4}$ does not qualify the parent-child relationship with $a_{7}$, the path $\left(a_{7}, b_{4}\right)$ is not output. The third call of getNextExt(root) returns $c_{9}$, and the path $\left(a_{7}, c_{9}\right)$ is output (line 10 in Algorithm 1). Similarly, the paths for $c_{10}$ and $c_{11}$ will both be output. But the twig pattern actually has zero result. The problem here is that we "wrongly" put $a_{7}$ onto stack, which does not have a qualifying $b$ child element at all! (Recall that we only knew that $a_{7}$ has a descendant $b_{4}$ when we pushed it) Then, is it possible to efficiently check the existence of one $b$ child before pushing $a_{7}$ ? The answer is, such a checking might be very costly because the first child of an element could be far away from its first descendant element.

The recent work by Choi et al [5] also provided some insight into the cause of the suboptimality in evaluating twig patterns with arbitrarily mixed ancestordescendant and parent-child edges.

\section{A performance study}

In this section, we present the experiments conducted to evaluate the effectiveness of various algorithms and heuristics proposed in the paper and report some of the results obtained.

\subsection{Experimental setup}

As Table 1 shows, with different combinations of choices in the dimensions of index and algorithm, we have many algorithms for twig joins. Here, we will focus on three kinds of algorithms, namely the TwigStack, TwigStackXB and XRTwig algorithms. The first two algorithms were chosen as they were the best twig join algorithms prior to this paper. We also implemented TSGeneric ${ }^{+}$with other kinds of indices, however, TSGeneric ${ }^{+}$with the XR-tree index, i.e., 
XRTwig, usually performs best. We have also implemented the three variants of XRTwig based on different edge-picking heuristics (top-down, bottom-up and maximum-distance), resulting in the XRTwig(TD), $X R T w i g(B U)$ and $X R T w i g(M D)$ algorithms respectively. We will later compare those algorithms from several perspectives.

Table 1: Diagrammatic view of algorithms

\begin{tabular}{|c||c|c|}
\hline Index Algo & TSGeneric & TSGeneric $^{+}$ \\
\hline \hline No Index & TwigStack & \\
\hline XB-tree & TwigStackXB & \\
\hline XR-tree & & XRTwig \\
\hline $\mathrm{B}^{+}$-tree & & \\
\hline R-tree & & \\
\hline
\end{tabular}

We evaluated the performance of those join algorithms using the following three metrics:

- number of elements scanned. We measure the total number of elements scanned during a join, which reflects the ability of each algorithm to skip elements that do not belong to the final result.

- number of page accesses. This metrics measures the performance of algorithms in terms of $\mathrm{I} / \mathrm{O}$ cost.

- running time. The running time of an algorithm is obtained by averaging the running times of several consecutive runs with hot buffers.

In this paper, we mainly report our results that demonstrate the performance of algorithms for data with different characteristics. We fixed a set of queries and executed those queries on different datasets, designed with different kinds of selectivity. Intuitively, a high selectivity (i.e., few matches) tends to favor algorithms utilizing indices.

Three query patterns were selected to represent different classes of twig patterns. They include a simple path query (Q1), a deep twig (Q2) and a bushy twig (Q3) as shown in Figure 5. All edges in the queries are ancestor-descendant relationships because all these algorithms deal with parent-child edges much the same way as ancestor-descendant edges, though, without guarantee of optimality.

We chose to generate synthetic datasets so that we can better control the relationship between the algorithms and the characteristics of the datasets. We generated 8 datasets for Q1, 10 for Q2 and 10 for Q3. There are two types of datasets: with varying selectivities and with the same selectivity. For example, we generated 4 datasets $D S_{i}, 1 \leq i \leq 4$, for Q1 in which the selectivities of different edges are different. For $D S_{1}$, the selectivity of the $A-B$ edge is $1 \%$ while the selectivity of the $B-C$ edge is $10 \%$. We used a "roundrobin" fashion method to generate other datasets by

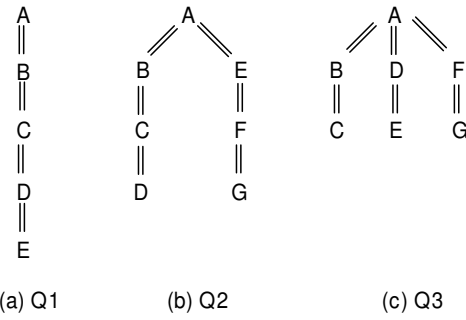

Figure 5: Three query patterns used

cyclically assigning the selectivity of the current edge to the next edge. For datasets of fixed selectivity, we simply assigned the same selectivity to all the edges. For example, all the edges of Q1 on dataset $D S_{5}$ have the same selectivity, i.e. $1 \%$.

Table 2 gives the description of datasets with varying selectivities for Q1. We also generated 4 datasets, $D S_{5}$ to $D S_{8}$, with fixed edge selectivity values of $1 \%$, $10 \%, 50 \%$ and $100 \%$ respectively.

Table 2: Selectivity of edges in Q1 for $D S_{1}$ to $D S_{4}$

\begin{tabular}{|c||r|r|r|r|}
\hline datasets & $A \triangleleft B$ & $B \triangleleft C$ & $C \triangleleft D$ & $D \triangleleft E$ \\
\hline \hline$D S_{1}$ & $1 \%$ & $10 \%$ & $50 \%$ & $100 \%$ \\
$D S_{2}$ & $10 \%$ & $50 \%$ & $100 \%$ & $1 \%$ \\
$D S_{3}$ & $50 \%$ & $100 \%$ & $1 \%$ & $10 \%$ \\
$D S_{4}$ & $100 \%$ & $1 \%$ & $10 \%$ & $50 \%$ \\
\hline
\end{tabular}

Since Q2 has six edges in the pattern, we used six different join selectivity values to generate six "selectivity round-robin" datasets, as shown in Table 3. Similarly as for Q1, four other datasets $D S_{7}$ to $D S_{10}$ were generated to test the case when all edges in Q2 have the same selectivity, i.e. $1 \%, 10 \%, 50 \%$ and $100 \%$ respectively. The 10 datasets for Q3 were similarly generated and the description is omitted in the interest of space.

Table 3: Selectivity of edges in Q2 for $D S_{1}$ to $D S_{6}$

\begin{tabular}{|c||r|r|r|r|r|r|}
\hline DS & $A \triangleleft B$ & $A \triangleleft E$ & $B \triangleleft C$ & $E \triangleleft F$ & $C \triangleleft D$ & $F \triangleleft G$ \\
\hline \hline$D S_{1}$ & $1 \%$ & $10 \%$ & $25 \%$ & $50 \%$ & $75 \%$ & $100 \%$ \\
$D S_{2}$ & $10 \%$ & $25 \%$ & $50 \%$ & $75 \%$ & $100 \%$ & $1 \%$ \\
$D S_{3}$ & $25 \%$ & $50 \%$ & $75 \%$ & $100 \%$ & $1 \%$ & $10 \%$ \\
$D S_{4}$ & $50 \%$ & $75 \%$ & $100 \%$ & $1 \%$ & $10 \%$ & $25 \%$ \\
$D S_{5}$ & $75 \%$ & $100 \%$ & $1 \%$ & $10 \%$ & $25 \%$ & $50 \%$ \\
$D S_{6}$ & $100 \%$ & $1 \%$ & $10 \%$ & $25 \%$ & $50 \%$ & $75 \%$ \\
\hline
\end{tabular}

Each element set generated contains $250 \mathrm{~K}$ elements. As a result, each dataset for Q1 involves more than one million elements while each dataset for Q2 and Q3 has near two million elements. Elements can be self-nested up to five levels. The join result size for each dataset varied according to the selectivity of edges. Take Q1 as an example. The numbers of path solutions for $D S_{1}$ to $D S_{4}$ are $29 \mathrm{~K}, 36 \mathrm{~K}, 38 \mathrm{~K}$ and $35 \mathrm{~K}$ respectively. There is no output solution when all edges are very selective ( $1 \%$ for $\left.D S_{5}\right)$ while there are more than 70 


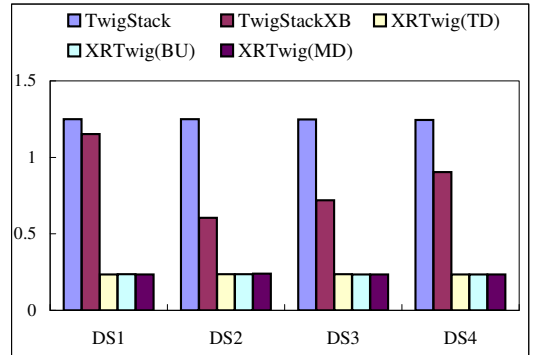

(a) \#elements scanned (million)

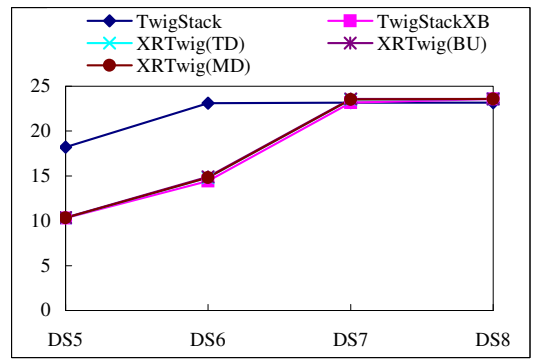

(d) \#page accesses (thousand)

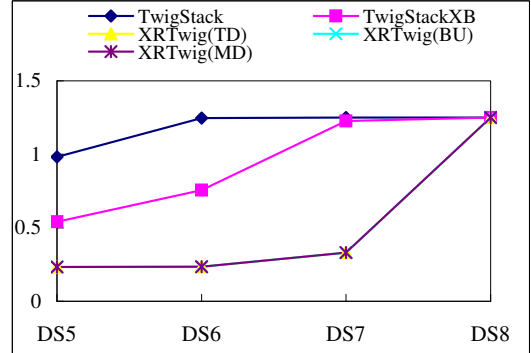

(b) \#elements scanned (million)

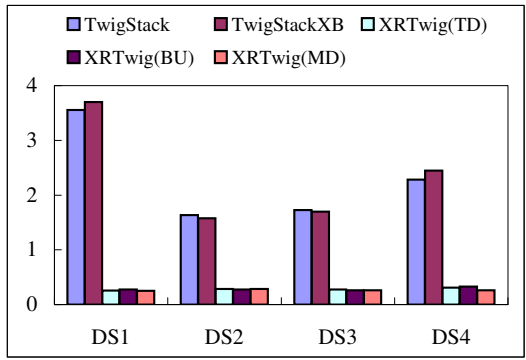

(e) running time (second)

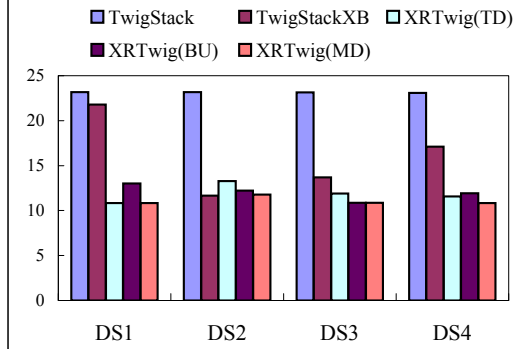

(c) \#page accesses (thousand)

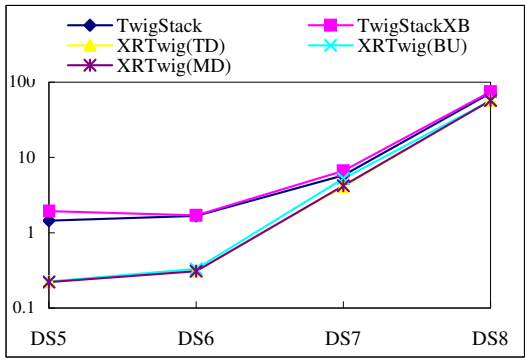

(f) running time (second)

Figure 6: Experimental results for query Q1

million path solutions when the selectivity of edges is very low $\left(100 \%\right.$ for $\left.D S_{8}\right)$.

Our test-bed is an experimental database system that includes a storage manager, a buffer pool manger, $\mathrm{B}^{+}$-tree, XB-tree and XR-tree index modules. All the algorithms were coded using Microsoft Visual $\mathrm{C}++$ 6.0. All the experiments were conducted on a Pentium IV $1.60 \mathrm{GHz}$ PC with 512M RAM and a $30 \mathrm{G}$ hard disk, running Windows XP. The page size used is $4 \mathrm{~K}$ and we used the file system as the storage. All the experimental results presented below were obtained with a fixed buffer pool size: 500 pages, just large enough to cache the hot paths.

Figure 6 shows all the results on Q1 for the five different algorithms/heuristics and 8 datasets. Figure 7 and 8 give the numbers of elements scanned and numbers of page accesses on Q2 and Q3 respectively (we ignore the results for TwigStack because it performed almost always worse than TwigStackXB as can be seen from Figure 6).

\subsection{Effects of using indices}

[3] reported experiment results of TwigStack and TwigStackXB. Our results coincide with theirs. Specifically, both the number of elements scanned and the number of page accesses for TwigStackXB are significantly smaller than those for TwigStack in most cases. For example, TwigStack scanned about 2 times as many elements as TwigStackXB for $D S_{2}$ on Q1 (See Figure 6(a)). This is simply because TwigStack always scans almost all the elements while TwigStackXB can identify and skip unmatched elements. On the other hand, under the extreme case where there is hardly any element that can be skipped, for example, the $D S_{8}$ on Q1, TwigStack performs the best, scanning the least number of elements and incurring the least amount of I/O. TwigStackXB will have some overhead due to the access to the internal pages of the index, although such overhead is negligible.

It is interesting to compare their performance, for example, in terms of the number of elements scanned, of $D S_{1}$ on Q1. For this dataset, the selectivity of edges changes from $1 \%$ to $100 \%$ from top to bottom. As a result, there is not much opportunity for TwigStackXB to skip elements through the fwdToAncestorOf operation (line 10 in Algorithm 3) because the operation is very frequently called due to the low selectivity of lower edges. On the contrary, for $D S_{2}$ for which the lowest edge has selectivity $1 \%$, the matches between $D$ and $E$ are rare, therefore, there is high potential for skipping elements in upper query nodes.

We conclude that twig join algorithms can enhance their performance consistently with the help of indices in most cases, without incurring noticeable overhead even for the worst case scenario.

\subsection{TSGeneric ${ }^{+}$vs. TSGeneric}

Now we compare the performance of all the algorithms based on TSGeneric ${ }^{+}$with the algorithm based on TSGeneric (i.e., TwigStackXB). Both classes of algorithms can make use of indices to skip some unmatched elements.

It can be observed that, for example, from experiment results on Q1 (Figure 6), TSGeneric ${ }^{+}$ based algorithms are usually much more efficient than TwigStackXB. For Q1 in terms of running time, 
$\square$ TwigStackXB $\square$ XRTwig(TD) $\quad$ XXRTwig(BU) $\quad$ 口XRTwig(MD)

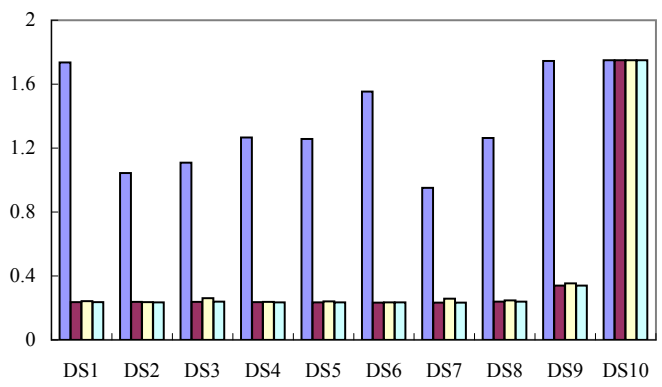

(a) \#elements scanned (million)

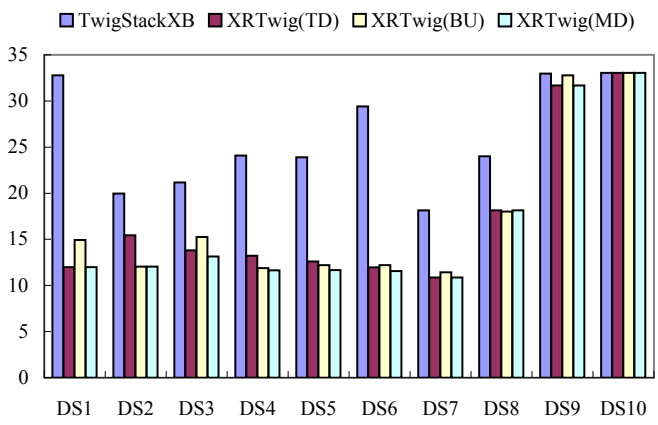

(b) \#page accesses (thousand)

Figure 7: Experimental results for query Q2

TSGeneric ${ }^{+}$based algorithms could be up to 10 times faster than TwigStackXB. Similar trends can be observed in other examples and in terms of other metrics as well. For example, we find that the advantage of TSGeneric ${ }^{+}$based algorithms over TwigStackXB is even greater for most datasets on Q2 and Q3. In particular, for $D S_{1}$ in terms of the number of elements scanned (see, Figure 7(a) and 8(a)), TSGeneric ${ }^{+}$only scanned less than $1 / 7$ of the total elements scanned by TwigStackXB. Meanwhile, in terms of the number of page accesses, TwigStackXB performed much worse than TSGeneric ${ }^{+}$, even for datasets with low selectivity $D S_{7}$ to $D S_{10}$ (see, Figure 7(b) and 8(b)), though it showed similar performance with TSGeneric ${ }^{+}$for those fixed selectivity datasets on Q1.

The performance advantage of TSGeneric ${ }^{+}$over TSGeneric mainly attributes to the fact that with a "pick-and-fix" strategy for locating solution extensions, TSGeneric ${ }^{+}$stands more chances of taking the advantage of edges with high selectivity to skip elements. Furthermore, for twig pattern queries, the cursor advances in one branch help more in TSGeneric ${ }^{+}$ than in TSGeneric to skip elements in another branch due to the unordered evaluation of edges in the extension location procedure of TSGeneric ${ }^{+}$.

Generally speaking, TSGeneric ${ }^{+}$has stronger capability to identify and skip unmatched elements, especially when the twig pattern is complex.

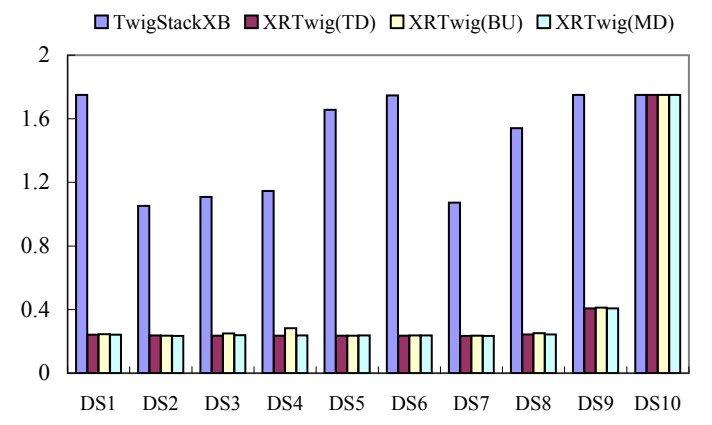

(a) \#elements scanned (million)

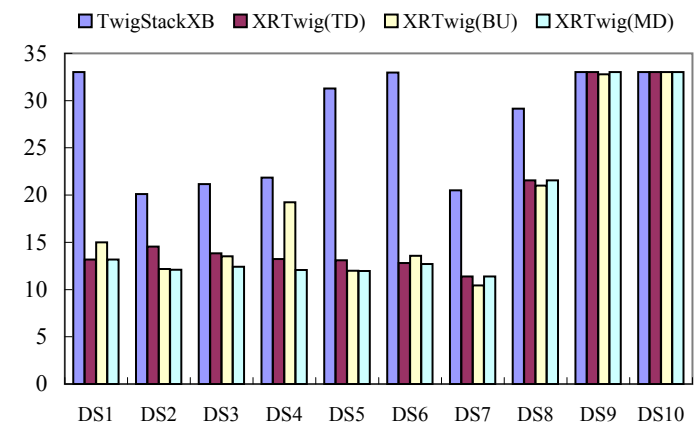

(b) \#page accesses (thousand)

Figure 8: Experimental results for query Q3

\subsection{Comparison of heuristics}

In this subsection, we study the three proposed heuristics for edge-picking. To that end, we also summarize, in Figure 9, the numbers of page accesses for all the queries with varying edge selectivities on Q1, Q2 and Q3.

$\square$ XRTwig(TD) $\square$ XRTwig(BU) $\square$ XRTwig(MD)

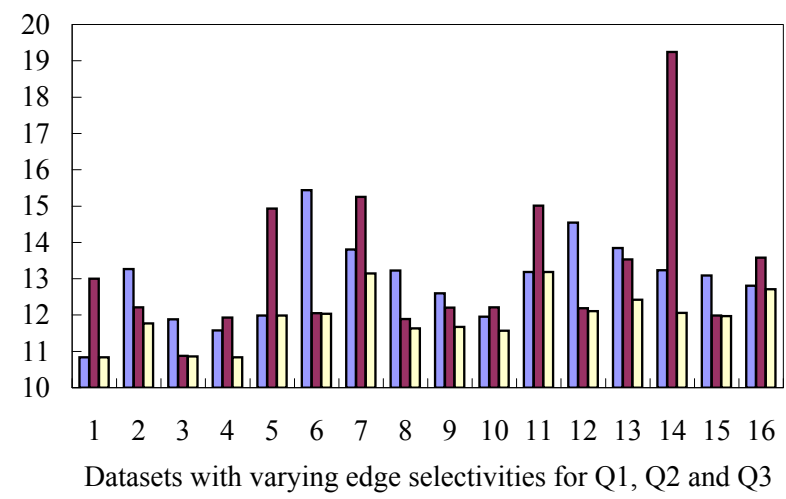

Figure 9: \#Page accesses under different edge-picking heuristics (thousand)

We can draw several interesting observations from Figure 6 to Figure 9:

- It is quite surprising that the difference between the three edge-picking heuristics of the TSGeneric ${ }^{+}$algorithm is not significant, in terms 
of the number of elements scanned (see, Figure 6(a), 6(b), 7(a) and 8(a)). This is mainly due to the fact that although MD always picks the most selective edge (with accurate estimates) to fix, other heuristics somehow also have the opportunity to select such an edge (after other high priority edges have been fixed).

- Despite their similar numbers of elements scanned, the three heuristics show various I/O performance (Figure 9). In particular, XRTwig(MD) has the best overall I/O performance, which indicates that, although XRTwig(MD) did not scan much less elements than algorithms based on the other heuristics, it managed to cluster those element scans better. For example, XRTwig(BU) for $D S_{4}$ on $\mathrm{Q} 3(\mathrm{X}=$ 14 in Figure 9) performed 1.6 times as much I/O as $X R T w i g(M D)$.

- The relative performance of $X R T w i g(T D)$ and $X R T w i g(B U)$ varies with the different formation of selectivities on the twig edges. Generally speaking, better I/O performance can be observed when the more selective edges have higher priority in terms of edge-picking heuristics. For example, XRTwig(TD) performed better than $X R T w i g(B U)$ for $D S_{1}$ and $D S_{4}$ on $\mathrm{Q} 1$, while $X R T w i g(B U)$ is better than $X R T w i g(T D)$ for $D S_{2}$ and $D S_{3}$ on Q1.

In summary, MD algorithm always performs the best as long as accurate statistics are available.

\section{Conclusions}

In this paper, we addressed the problem of efficient evaluation of holistic twig joins on all/partly indexed XML documents. In particular, we proposed TSGeneric ${ }^{+}$with three different evaluation heuristics, namely, top-down, bottom-up and statistics-based. Experimental results indicated that the TSGeneric ${ }^{+}$ algorithm on XR-tree indexed datasets performs significantly better than the existing ones by much more effectively skipping elements that do not contribute to final results, especially when binary structural joins in the twig pattern have varying selectivities. Among the three heuristics we considered, the statistics-based heuristic is most robust, given that the statistics used are accurate. As such, existing work on join selectivity estimation for XML data can be leveraged.

Regarding our future work, we will investigate new heuristics for the edge picking process in TSGeneric ${ }^{+}$, which may require more sophisticated estimation techniques for XML data. Another future work is to consider more complex queries, like a query pattern consisting of multiple twig patterns.

\section{References}

[1] A. Berglund, S. Boag, D. Chamberlin, M. F. Fernandez, M. Kay, J. Robie, and J. Simeon. XML path language (XPath) 2.0. Technical report, W3C, 2002.

[2] S. Boag, D. Chamberlin, M. F. Fernandez, D. Florescu, J. Robie, and J. Simeon. XQuery 1.0: An XML query language. Technical report, W3C, 2002.

[3] N. Bruno, D. Srivastava, and N. Koudas. Holistic twig joins: Optimal XML pattern matching. In SIGMOD, pages 310-321, 2002.

[4] S.-Y. Chien, Z. Vagena, D. Zhang, V. Tsotras, and C. Zaniolo. Efficient structural joins on indexed XML documents. In $V L D B$, pages 263-274, 2002.

[5] B. Choi, M. Mahoui, and D. Wood. On the optimality of holistic algorithms for twig queries. In DEXA, 2003.

[6] D. Florescu and D. Kossmann. Storing and querying XML data using an RDBMS. IEEE Data Engineering Bulletin, 22(3):27-34, 1999.

[7] T. Grust. Accelerating XPath location steps. In $S I G$ MOD, pages 109-120, 2002.

[8] H. Jiang, H. Lu, W. Wang, and B. C. Ooi. XR-Tree: Indexing XML data for efficient structural joins. In ICDE, pages 253-264, 2003.

[9] Q. Li and B. Moon. Indexing and querying XML data for regular path expressions. In $V L D B$, pages $361-370$, 2001.

[10] J. McHugh and J. Widom. Query optimization for XML. In $V L D B$, pages 315-326, 1999.

[11] K. Ono and G. M. Lohman. Measuring the complexity of join enumeration in query optimization. In $V L D B$, pages 314-325, 1990.

[12] P. G. Selinger, M. M. Astrahan, D. D. Chamberlin, R. A. Lorie, and T. G. Price. Access path selection in a relational database management system. In SIGMOD, pages 23-34, 1979.

[13] J. Shanmugasundaram, K. Tufte, C. Zhang, H. Gang, D. J. DeWitt, and J. F. Naughton. Relational databases for querying XML documents: Limitations and opportunities. In $V L D B$, pages 302-314, 1999.

[14] D. Srivastava, S. Al-Khalifa, H. V. Jagadish, N. Koudas, J. M. Patel, and Y. Wu. Structural joins: A primitive for efficient XML query pattern matching. In $I C D E$, pages 141-152, February 2002.

[15] I. Tatarinov, S. Viglas, K. Beyer, J. Shanmugasundaram, E. Shekita, and C. Zhang. Storing and querying ordered XML using a relational database system. In SIGMOD, pages 204-215, 2002.

[16] B. Vance and D. Maier. Rapid bushy join-order optimization with cartesian products. In SIGMOD, pages 35-46, 1996.

[17] W. Wang, H. Jiang, H. Lu, and J. X. Yu. Containment join size estimation: Models and methods. In SIGMOD, pages 145-156, 2003.

[18] Y. Wu, J. M. Patel, and H. V. Jagadish. Estimating answer sizes for XML queries. In EDBT, pages 590 608, March 2002.

[19] Y. Wu, J. M. Patel, and H. V. Jagadish. Structural join order selection for XML query optimization. In ICDE, pages 443-454, March 2003.

[20] C. Zhang, J. F. Naughton, D. J. DeWitt, Q. Luo, and G. M. Lohman. On supporting containment queries in relational database management systems. In $S I G$ MOD, pages 425-436, 2001. 